\title{
Studying Materials and Processes with VISION, VirtuES and ICEMAN - Modeling INS Data with DFT Methods
}

\author{
Anibal J Ramirez-Cuesta and Yongqiang (YQ) Cheng \\ Neutron Science Directorate, Oak Ridge National Laboratory, Oak Ridge, TN, United States.

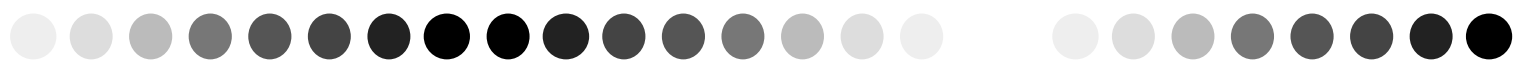

\begin{abstract}
Inelastic Neutron scattering (INS) is a very powerful tool to study the vibrational dynamics of solids. The VISION spectrometer at the SNS in Oak Ridge Tennessee has an increased overall flux at low energy transfers up to 100 times over its predecessors and it has unprecedented sensitivity. We will examine the limits of what is now possible in INS thanks to VISION. From the determination of INS spectra of publishable quality in minutes (for samples in the gram quantity range), measuring the signal of samples in the milligram range to the direct determination of the signal of $2 \mathrm{mmol}$ of $\mathrm{CO}_{2}$ adsorbed on functionalized catalysts. Finally, the major challenges that we are facing will be discussed, in particular methods to automate data analysis and interpretation through computer modelling and Artificial Intelligence/Machine Learning etc.
\end{abstract} \\ Keywords: Inelastic Neutron Scattering, Computer Modeling, Data Analysis
}

\section{Introduction}

The VISION spectrometer is located on beamline $16 \mathrm{~b}$ (BL 16b) at the Spallation Neutron Source (SNS) in Oak Ridge Tennessee. VISION is quite unique since data analysis, in most cases, requires the use of DFT modeling and software to convert these computer models into synthetic spectra that can be directly compared with experimental data.

VISION is an indirect geometry inelastic neutron scattering spectrometer that boasts the highest flux and resolution for instruments of its kind. The primary flightpath is $16 \mathrm{~m}$ from a decoupled water moderator at ambient temperature [1]. The secondary flightpath is $0.73 \mathrm{~m}$. The secondary spectrometer, depicted in Fig. 1 has an analyzer that consists of a parametric array of 347 single crystal pyrolytic graphite (PG 002) crystals $1 \mathrm{~cm}^{2}$ each, that focusses the scattered beam into a small area on ${ }^{3} \mathrm{He}$ tubes. Between the analyzer and the detectors there is a sliced Be block with $\mathrm{Cd}$ sheets separating the wedges. These Be filters remove the unwanted $\lambda / n$ reflections from the crystal analyzers, operating as bypass filters. The total energy transfer range is from $-2 \mathrm{meV}$ to $1000 \mathrm{meV}$, and it goes across the elastic line. The instrumental resolution for an instrument of this kind, for energy transfers above 5 $\mathrm{meV}$ is almost constant as a fraction of the energy transfer [2]:

$$
\Delta \omega / \omega \sim 1.5 \%
$$

At the elastic line the resolution is $120 \mu \mathrm{eV}$.
There is a backscattering diffraction bank and six diffraction banks located at 90 degrees. So, the VISION instrument can simultaneously measure inelastic, quasielastic and diffraction. It is a true multimodal instrument.

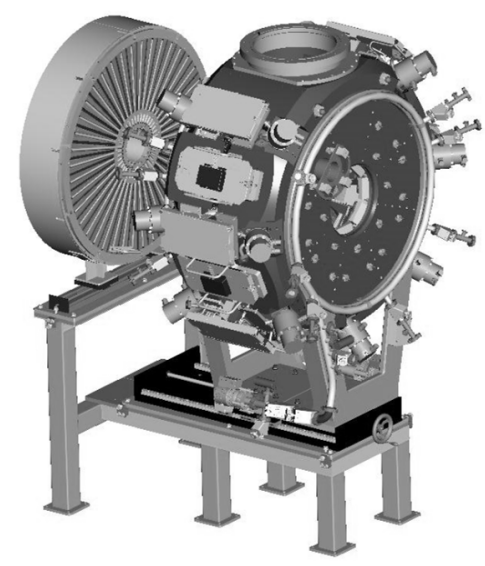

Fig. 1. Engineering drawing of VISION. On the left the backscattering diffraction bank, the body of the instrument in the center houses the inelastic analyzers, filters, detectors and the 90 degrees diffraction banks.

2. Computer modeling as part of the instrument

As mentioned before, the inelastic cross section can be rigorously calculated in the case of solids. We have developed software that can produce synthetic spectra from ab-initio calculations $[3,4]$.

Simulation of INS is, in theory, straightforward. There is a rigorous theory established for the 
calculation of INS spectra from phonons (normal modes frequencies and polarization vectors). Albeit trivial for fundamental excitations, the extension and application for higher order combinations and overtones is not quite simple. The most advanced software to date is OCLIMAX [4]. The output from computer models provides the information on phonons. It can be obtained from either quantum or classical calculations. The OCLIMAX software includes experimental instrumental features into consideration, such as the instrument geometry, resolution, nature of the sample, etc. This kind of modeling is crucial connecting theory and experiment, and in many cases interpretation of the experimental data is just not possible without them.

The calculations required for many materials are quite demanding. The VirtuES cluster is dedicated to VISION data analysis and modeling, it has 32 cores per node, 50 compute nodes, making 1600 (nonhyperthreaded) cores; it is installed as part of the ORNL Compute and Data Environment for Science (CADES). VirtuES is considered part of the VISION instrument.

3. Instrument sensitivity, high throughput, small samples

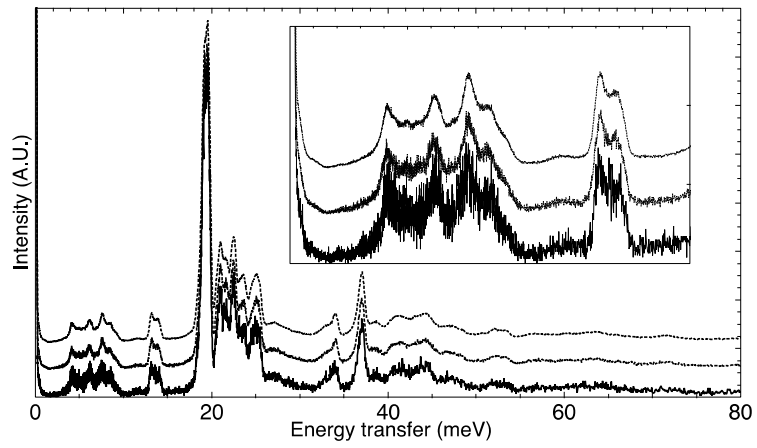

Figure 2. The spectra of M-POSS with different collection times. Bottom trace $25 \mathrm{~s}$, middle trace $4 \mathrm{~min}$ and top trace 15 $\min$.

VISION has extraordinary sensitivity, it can measure a $1 \mathrm{~g}$ size sample to publication quality in a few minutes. Polyoligosilsesquioxanes (POSS) are molecules composed of a silicon-oxygen cage $\left(\mathrm{Si}_{8} \mathrm{O}_{12}\right)$ in which, for example, $\mathrm{Si}$ atoms occupy the corners of a cube and oxygen atoms are located on the edges. Each $\mathrm{Si}$ atom is bonded to three oxygen atoms but may have any one of a wide variety of potential ligands attached to the fourth position. POSS molecules can also be viewed as very small, perfectly monodisperse silica nanoparticles, with typical diameters on the order of 1-2 $\mathrm{nm}$. These can be considered the smallest possible silica nanoparticles, with sizes that fall between typical solvent molecules (diameters of $0.2-0.5 \mathrm{~nm}$ ) and the smallest commercially available. Jalarvo and corworkers reported the results of a comprehensive study of the structure and dynamics of the octamethylPOSS (M-POSS) molecule $\mathrm{Si}_{8} \mathrm{O}_{12}\left(\mathrm{CH}_{3}\right)_{8}$, where the eight ligands are methyl groups [5]. During the experiment the data was analyzed for different collection times, Figure 2. It can be seen that a spectrum of publishable quality is available after 4 minutes of data collection time.

The fast measurement in the above case for $1 \mathrm{~g}$ of $\mathrm{M}$ POSS also means that VISION can measure very small amount of sample. To test this capability, we measured sucrose (table sugar) and gradually reduced the amount of the sample in the beam (Figure 3). It is found that even with only a few grains of sugar $\left(1.25 \mathrm{mg}\right.$, or $\left.0.8 \mathrm{~mm}^{3}\right)$, it is still possible to observe the main features of the spectrum given longer data collection time.

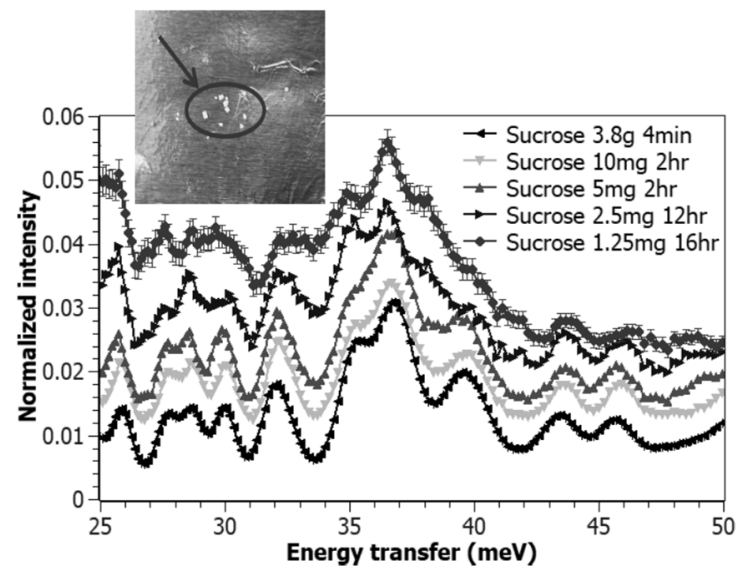

Figure 3. Sucrose (table sugar) measured at VISION. The sample amount decreases from the bottom spectrum (3.8g) to the top spectrum $(1.25 \mathrm{mg})$. Inset shows a picture of the $1.25 \mathrm{mg}$ sample.

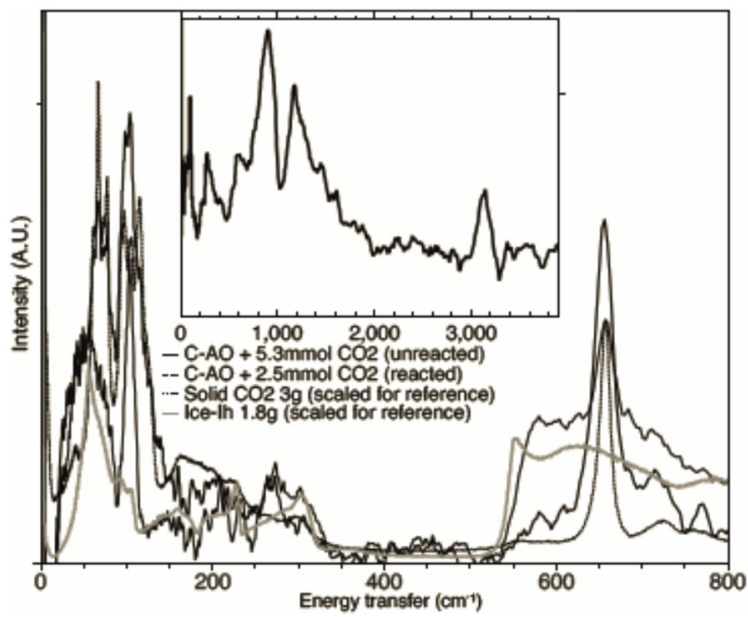

Figure 4. The difference INS spectra before and after $\mathrm{CO}_{2}$ dosing in $\mathrm{C}-\mathrm{AO}$, in comparison with the reference spectra for bulk solid $\mathrm{CO}_{2}$ and $\mathrm{H}_{2} \mathrm{O}$. Signal from the background and the blank $\mathrm{C}-\mathrm{AO}$ has been subtracted. Inset shows the INS spectrum of the blank $\mathrm{C}$-AO before dosing (note that the energy scale is different) [6]. 
Another example of the sensitivity for small signal is the study of non-hydrogenous gas adsorption on a carbon substrate. $\mathrm{CO}_{2}$ interactions with nanoporous $\mathrm{S}$ and $\mathrm{N}$-doped polymer-derived carbon and commercial wood-based carbon were investigated in a broad range of conditions. The results showed that during $\mathrm{CO}_{2}$ adsorption nitrogen and sulfur species as well as water were released from the carbon surface as a result of chemical reactions of the surface groups with $\mathrm{CO}_{2}$. Inelastic neutron scattering experiments provided the unprecedented ability to characterize very small amounts of $\mathrm{CO}_{2}$ and $\mathrm{H}_{2} \mathrm{O}$ and revealed for the first time their physical/chemical status in the confined space of nanoporous carbons. The results obtained suggest that the reactivity of the carbon surface should be considered when $\mathrm{CO}_{2}$ storage media are chosen and when $\mathrm{CO}_{2}$ is used as a probe to determine the microporosity of carbon materials [6]. The instrument is able to measure the small signal difference between the empty sample and the sample with $5 \mathrm{mmol}$ of $\mathrm{CO}_{2}$, Fig. 4.

4. Studying gas adsorption with VISION and modeling

Atomistic modeling is an essential part of data analysis and interpretation at VISION, and such an integrated approach has been used to study a large variety of physical and chemical systems. For example, metal-organic frameworks (MOFs) have important applications for gas storage, separation, and catalysis. To understand how MOFs work for these applications, it is crucial to understand the interaction between the guest molecules and the MOFs (i.e., the metal sites and/or the organic ligands). Neutron spectroscopy can be particularly useful for such studies. In a recent paper published in Nature Materials [7], researchers from University of Manchester used VISION to study $\mathrm{NO}_{2}$ adsorption on an Al-MOF, named MFM-300. This MOF is demonstrated to have reversible and recordhigh $\mathrm{NO}_{2}$ uptake, and therefore can be a great material for pollutant removal from the air. The INS spectra of MFM-300 before and after $\mathrm{NO}_{2}$ adsorption were measured, and small changes in the spectra can be observed upon $\mathrm{NO}_{2}$ adsorption (Fig. 5a, top panel).

However, it would be virtually impossible to interpret the differences without advanced modeling tools. Using the dedicated computational resources available for VISION, simulations can be performed for the bare and $\mathrm{NO}_{2}$-dosed MOF. It is found that the main features in spectra are well reproduced, thus all the major peaks can be assigned to their corresponding vibrational modes (Fig. 5a, bottom panel). What is captured even better is the difference spectrum, i.e. the spectrum of the loaded MOF minus the spectrum of the bare MOF (Fig. 5b). Since $\mathrm{NO}_{2}$ itself has very low neutron scattering cross-section compared to hydrogen in the organic ligands, the difference spectrum mainly reflects the changes of the ligand vibrations due to ligand- $\mathrm{NO}_{2}$ interactions. Specifically, in the intermediate energy range, blue shift can be clearly seen for peaks III to VI. These peaks correspond to the outof-plane bending of $\mathrm{OH} / \mathrm{CH}$ (III and IV), and in-plane bending of $\mathrm{OH} / \mathrm{CH}(\mathrm{V}$ and $\mathrm{VI})$, and they are local dynamics featured by small atomic displacements.

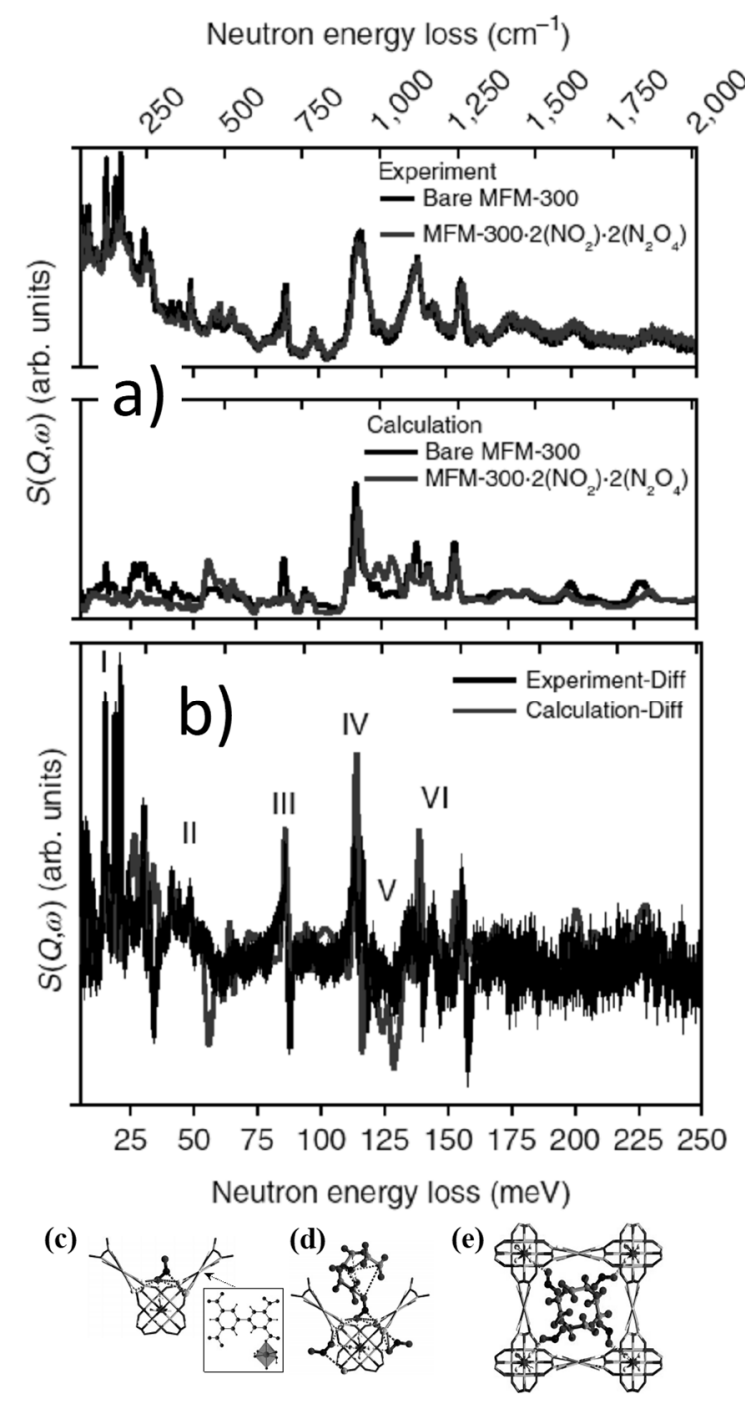

Figure 5. (a) VISION spectra of bare MFM-300 and $\mathrm{NO}_{2}$ loaded MFM-300 (top: experiment, bottom: simulation). (b) Measured and simulated difference spectra. (c) Local configuration of the anchor $\mathrm{NO}_{2}$ monomer (inset shows the side view of the organic ligands). (d) Local configuration including the $\mathrm{N}_{2} \mathrm{O}_{4}$ dimmers. (e) Full unit cell of the $\mathrm{MOF}+\mathrm{NO}_{2}$ structure. Reproduced from Ref [7]. Copyright Nature Publishing Group 2018.

Their blue shift can be attributed to the hydrogen bonding and charge redistribution between the anchor $\mathrm{NO}_{2}$ monomer and the $\mathrm{OH}$ on $\mathrm{Al}$ and the $\mathrm{CH}$ on the ligand rings (Fig. 5c). On the other hand, the low energy peaks (I and II) are mainly seeing intensity reductions. These peaks can be assigned to the out-of-plane (I) and in-plane (II) twisting of the ligand rings. These lowenergy modes are collective dynamics with relatively large atomic displacements, and the intensity reduction is caused by the constrained motion of the rings due to 
the presence of the $\mathrm{N}_{2} \mathrm{O}_{4}$ dimmers that fill up the pores (Fig. 5d). The spectroscopic evidence of $\mathrm{NO}_{2}-\mathrm{MOF}$ interactions and the atomistic insight, as detailed above, would not have been acquired without the modeling capabilities at VISION.

\section{Diffraction capabilities of VISION}

The VISION instrument is not only a spectrometer; it also measures diffraction simultaneously. The diffraction banks make use of the scattered neutrons that are not covered and collected by the inelastic banks. In other words, these neutrons are scattered by the same sample, under the same sample environment, and at the same time when the INS is measured. It is therefore truly in situ and simultaneous. The time-of-flight (TOF) diffraction data can be analyzed following standard refinement procedures, as implemented in various programs such as GSAS-II [8], FullProf [9], and TOPAS [10]. An example of $\mathrm{BaTiO}_{3}$ Rietveld refinement with GSAS-II can be seen in Fig. 6. The 90degree diffraction banks at VISION cover $Q$ range from 1.2 to $60 \AA^{-1}$, corresponding to a d spacing from 0.1 to $5.4 \AA$. The resolution $\Delta \mathrm{d} / \mathrm{d}$ is about $1 \%$.

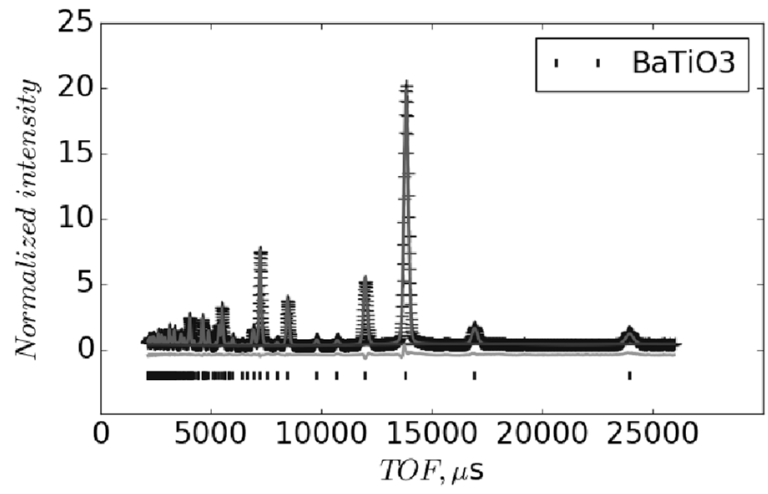

Figure 6. Rietveld refinement of VISION diffraction data on BaTiO3, using GSAS-II.

\section{Quasielastic and inelastic study with VISION}

This work presents an INS investigation of molecular hydrogen confined in microporous carbon. The nature of the interaction potential between $\mathrm{H}_{2}$ and adsorption sites has been probed by monitoring the para to ortho transition as a function of temperature, pressure, and $\mathrm{H}_{2}$ loading.

The state of molecular hydrogen, when adsorbed in porous materials, is a matter of controversy. Solid $\mathrm{H}_{2}$ behaves as a three-dimensional quantum rotor with energy levels given by:

$$
E_{J}=B J(J+1)
$$

where, $J$ is the quantum rotational number $(J=0,1, \ldots)$, and $B$ is the rotational constant of hydrogen defined as:

$$
B=\hbar^{2} /\left(2 \mu d_{e}^{2}\right)=7.35 \mathrm{meV}
$$

where $\mu$ is the reduced mass of the molecule, and $d_{e}$ is the equilibrium distance between the two protons $(0.741 \AA$ in the free state) $[2,11]$. There are two nuclear- spin isomers of molecular $\mathrm{H}_{2}$, namely ortho and para, differ by the parity of rotational quantum number, as required by the total wave function symmetry with respect to the permutation of the two nuclei. The states with even $J$ values are called para-hydrogen $\left(\mathrm{p}-\mathrm{H}_{2}\right)$ while those with odd $J$ values are called ortho-hydrogen $\left(\mathrm{o}-\mathrm{H}_{2}\right)$. Neutrons have spin and their interaction with $\mathrm{H}_{2}$ molecules (i.e., the INS) it has been known [12] that the transitions observed are $J_{(0 \rightarrow 1)}$ para to ortho hydrogen, a line at $14.7 \mathrm{meV}$ in the solid, and if there is ortho hydrogen present the $J_{(1 \rightarrow 1)}$ ortho to ortho hydrogen combined with quasielastic and inelastiuc signal. It is important to note that the $J_{(0 \rightarrow 0)}$ para to parahydrogen transition has very low cross section and it is almost invisible.

In the case of liquid para hydrogen, the rotational line of parahydrogen is not present in the spectrum because the molecule recoils. The quasielastic signal is present when ortho hydrogen is liquid or solid, and the total amount of hydrogen, solid, liquid and gas is present when the signal is integrated over the whole spectrum.

At $77 \mathrm{~K}$ molecular hydrogen is supercritical, so it cannot be a solid under pressure under 5 kbar [13].

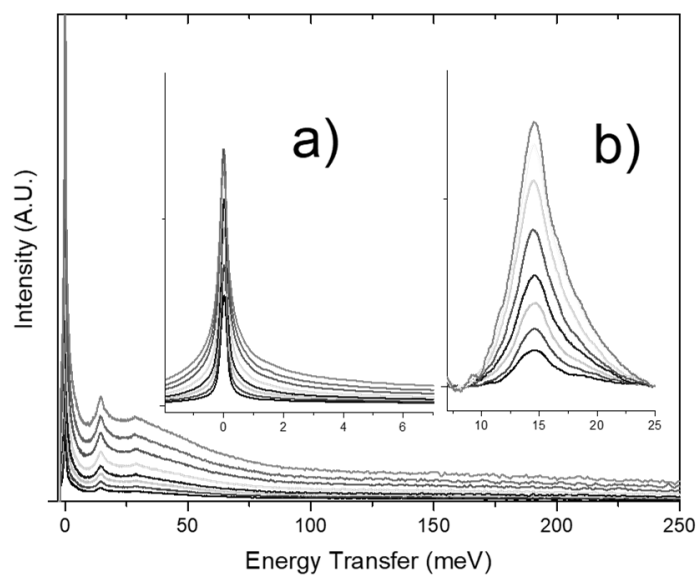

Figure 7. Evolution of elastic/quasielastic signal (a) and rotor peak (b) as a function of pressure $(0.10,0.23,0.51,1.03,3.25$, $11.2,22.4$ and 40.0 bar) at $77 \mathrm{~K}$, Recreated from data from Ref [13].

The data in Figure 7 clearly shows the increase in the QENS signal with pressure, as well as the rotational peak. Integrating the inelastic peak (rotor peak), the QENS peak and the total intensity we can observe the densification of the hydrogen in the pores as function of pressure, as shown in Figure 8. The presence of solid-like (immobile) and liquid-like (partially mobile) $\mathrm{H}_{2}$ was determined over a broad range of pressures and temperatures, including high pressure (up to 40 bar) and supercritical temperatures (up to $110 \mathrm{~K}$ ) [13]. 


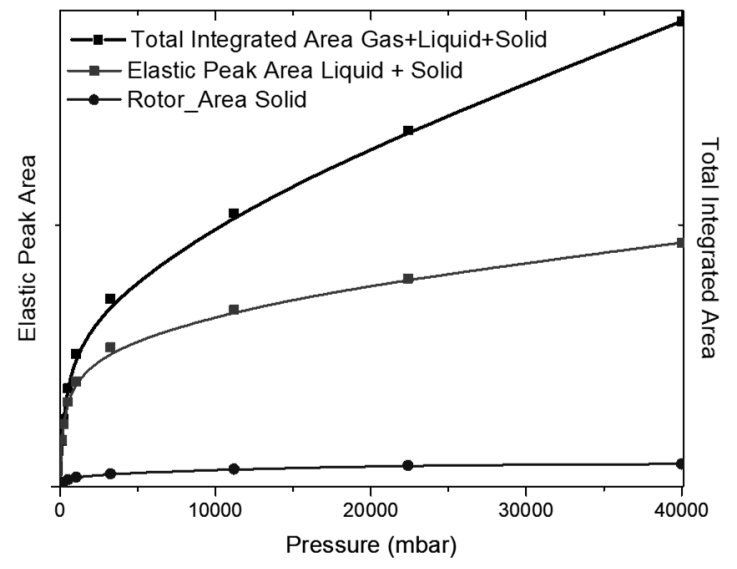

Figure 8. Comparison between $\mathrm{H}_{2}$ excess adsorption, deconvoluted elastic and quasielastic signals, and total INS signal as a function of pressure at $77 \mathrm{~K}$. Redrawn from Ref [13].

\section{Conclusions and outlook}

The VISION spectrometer at the SNS is a remarkable neutron instrument, truly multimodal, highly sensitive and with modeling capabilities tailored to users' requirements for data interpretation, including integrated computing resources such as the VirtuES cluster, ICEMAN software package, and various DFT programs.

The reduction and analysis are semi-automated. Albeit the data can be manually analyzed using the Mantid software [14], more advanced analysis is done using a series of semiautomated python scripts that are usually prepared by the instrument scientists and provided to the user.

To take further advantage of the link between models and experiments, new developments will be required for the future. In particular the analysis will need to include the use of modern computational methodologies, nowadays loosely called artificial intelligence (AI) or machine learning (ML). By using these techniques, we will be able to create semiautonomous software tools that will guide the user to understand and interpret their results faster.

\section{Acknowledgments}

The authors would like to thank Luke Daemen of VISION for their extensive advice and expertise. This research benefited from the use of the VISION beamline (IPTS-16527) at the Spallation Neutron Source, Oak Ridge National Laboratory (ORNL), which is supported by the Scientific User Facilities Division, Office of Basic Energy Sciences, US Department of Energy (DOE). OClimax is a part of the Integrated Computational Environment Modeling and Analysis of Neutron Data (ICE-MAN) (LDRD 8237) project, funded by the Laboratory Directed Research and Development program at ORNL.

\section{References}

[1] https://neutrons.ornl.gov/vision.

[2] P. C. H. Mitchell, S.F. Parker, A. J. Ramirez-Cuesta, J. Tomkinson, Vibrational spectroscopy with neutrons, Series on, World Scientific Publishing, Singapore, (2005).

[3] A. J. Ramirez-Cuesta, Comput. Phys. Commun. 157, 226 (2004).

[4] Y. Q. Cheng, L. L. Daemen, A. I. Kolesnikov, A. J. Ramirez-Cuesta, J. Chem. Theory Comput., 15, 3, (2019).

[5] N. Jalarvo, O. A. Gourdon, G. Ehlers, M. Tyagi, S. K. Kumar, K. D. Dobbs, R. J. Smalley, W. E. Guise, A. J. Ramirez-Cuesta, C. Wildgruber, M. K. Crawford, J. Phys. Chem. C, 118(10), 5579 (2014).

[6] T. J. Bandosz, M. Seredych, E. Rodriguez-Castellon, Y. Q. Cheng, L. L. Daemen, A. J. Ramirez-Cuesta, Carbon, 96, 856 (2016).

[7] Xue Han, Harry G. W. Godfrey, Lydia Briggs, Andrew J. Davies, Yongqiang Cheng, Luke L. Daemen, Alena M. Sheveleva, Floriana Tuna, Eric J. L. McInnes, Junliang Sun, Christina Drathen, Michael W. George, Anibal J. Ramirez-Cuesta, K. Mark Thomas, Sihai Yang, Martin Schröder, Nature Materials 17, 691 (2018).

[8] B. H. Toby, and R. B. Von Dreele, Journal of Applied Crystallography, 46(2), 544 (2013).

[9] Juan Rodríguez-Carvajal, Physica B: Condensed Matter, 192, 55 (1993).

[10] Alan A. Coelho, Journal of Applied Crystallography 51, 210 (2018).

[11] I. F. Silvera, Rev. Mod. Phys. 52, 393 (1980).

[12] J. A. Young and J. U. Koppel, Phys. Rev. 135, A603 (1964).

[13] J. Bahadur, C. I. Contescu, A. J. Ramirez-Cuesta, E. Mamontov, N. Gallego, Y. Q. Cheng, L. L. Daemen, Y. B. Melnichenko, Carbon 117, 383 (2017).

[14] O. Arnold, J. C. Bilheux, J. M. Borreguero, A. Buts, S. I. Campbell, L. Chapon, M. Doucet, N. Draper, R. Ferraz Leal, M. A. Gigg, V. E. Lynch, A. Markvardsen, D. J. Mikkelson, R. L. Mikkelson, R. Miller, K. Palmen, P. Parker, G. Passos, T. G.Perring, P. F. Peterson, S. Ren, M. A. Reuter, A. T.Savici, J. W.Taylor, R. J. Taylor, R. Tolchenov, W. Zhou, J. Zikovsky, Nucl. Instrum. Methods Phys. Res., Sect. A 764, 156 (2014). 
This list has been removed from the online version for privacy protection. 